\title{
Inteligencia emocional y desempeño laboral de los profesionales de la salud de un hospital de Pucallpa
}

\section{Emotional intelligence and work performance of the health professionals of the hospital of Pucallpa}

\section{Inteligência emocional e desempenho profissional de profissionais de saúde em um hospital de Pucallpa}

\author{
Eldalaine Torres Vargas \\ eldalaine_torres@unu.edu.pe \\ ORCID: 0000-0002-6786-0975
}

Miguel Coral Cevillano
mcoral@unamad.edu.pe
ORCID: 0000-0003-0889-4769

\author{
Nicolás Magno Fretel Quiroz \\ nfretel@unamad.edu.pe \\ ORCID: 0000-0002-2724-8116
}

\author{
Isabel Ramírez Chumbe \\ isabel_ramirez@unu.edu.pe \\ ORCID: 0000-0002-8936-9212
}

Universidad Nacional Amazónica de Madre de Dios, Puerto Maldonado-Perú

Recibido 14 de octubre 2020 | Arbitrado y aceptado 2 de noviembre 2020 | Publicado en 04 de enero 2021

\section{RESUMEN}

El Objetivo general de esta investigación fue el de establecer la relación entre Inteligencia Emocional y Desempeño Laboral de los profesionales de la salud de un Hospital de Pucallpa. La metodología utilizada fue de tipo descriptiva de corte transversal cuya muestra estuvo conformada por 125 elementos, 70 de sexo femenino y 55 de sexo masculino. Del total (125) elementos, 84 (67.2\%) poseen inteligencia emocional medio, $35(28.0 \%)$ inteligencia emocional alto, y $06(4.8 \%)$ inteligencia emocional bajo. Respecto al desempeño laboral, 88 (70.4\%) tienen Buen desempeño, $24(19.2 \%)$ un desempeño Excelente, y 13 (10.4\%) Deficiente desempeño. Como conclusión En base a los resultados obtenidos es evidente que la inteligencia emocional tiene una estrecha relación con el desempeño laboral de los profesionales de la salud puesto que para lograr un buen desenvolvimiento de sus actividades y poder cumplir con los objetivos de su cargo debe poseer la capacidad emocional de sobrellevar los retos y complicaciones que se le susciten a lo largo de su jornada laboral. Se evidencia que la inteligencia emocional se relaciona con el desempeño laboral, al someter al estadístico de prueba de Tau b de Kendall, con un nivel de confianza del $95 \%$ y grado de error del $5 \%$, se obtuvo un valor $\mathrm{p}=0.01$, lo que significa que existe relación estadísticamente significativa entre las variables.

Palabras clave: Inteligencia emocional; desempeño laboral; profesional

\begin{abstract}
The general objective of this research was to establish the relationship between Emotional Intelligence and Work Performance of the health professionals of a Hospital in Pucallpa. The methodology used was descriptive, cross-sectional, whose sample consisted of 125 elements, 70 female and 55 male. Of the total (125) elements, 84 (67.2\%) have medium emotional intelligence, 35 (28.0\%) high emotional intelligence, and $06(4.8 \%)$ low emotional intelligence. Regarding job performance, 88 (70.4\%) have Good performance, 24 (19.2\%) Excellent performance, and 13 (10.4\%) Poor performance. In conclusion Based on the results obtained, it is evident that emotional intelligence has a close relationship with the work performance of health professionals since to achieve a good development of their activities and to be able to fulfill the objectives of their position they must possess the emotional capacity to cope with the challenges and complications that arise throughout their workday. It is evidenced that emotional intelligence is related to job performance, when submitting to Kendall's Tau b test statistic, with a confidence level of $95 \%$ and a degree of error of $5 \%$, a value $p=0.01$ was obtained, which which means that there is a statistically significant relationship between the variables.
\end{abstract}

ET: Licenciada en Enfermería de la Universidad Nacional de Ucayali. Magister en Administración y Gerencia en Salud de la Universidad Nacional Hermilio Valdizan. Doctor en Ciencias de la Salud. Universidad Nacional de Ucayali, Perú.

NF: Licenciado en Enfermería. Magister en Educación, especialidad Investigación y Docencia Universitaria. Doctor en Administración de la Educación. Universidad Nacional Amazónica de Madre de Dios, Perú.

MC: Licenciado en enfermería de la MC: Licenciado en enfermería de la
Universidad Alas peruanas, Perú. Universidad Alas peruanas, Perú. Madre de Dios, Perú.

IR: Licenciada en Enfermería de la Universidad Nacional de Ucayali, Perú. 
ET: Licenciada en Enfermería de la Universidad Nacional de Ucayali. Magister en Administración y Gerencia en Salud de la Universidad Nacional Hermilio Valdizan. Doctor en Ciencias de la Salud. Universidad Nacional de Ucayali, Perú.

NF: Licenciado en Enfermería. Magister en Educación, especialidad Investigación y Docencia Universitaria. Doctor en Administración de la Educación. Universidad Nacional Amazónica de Madre de Dios, Perú.

MC: Licenciado en enfermería de la Universidad Alas peruanas, Perú Universidad Nacional Amazonica Madre de Dios, Perú.

IR: Licenciada en Enfermería de la Universidad Nacional de Ucayali, Perú.

\section{RESUMO}

0 objetivo geral desta pesquisa foi estabelecer a relação entre Inteligência Emocional e Desempenho no Trabalho dos profissionais de saúde de um Hospital de Pucallpa. A metodologia utilizada foi descritiva, transversal, cuja amostra foi composta por 125 elementos, sendo 70 do sexo feminino e 55 do sexo masculino. Do total (125) elementos, $84(67,2 \%)$ possuem média inteligência emocional, 35 $(28,0 \%)$ alta inteligência emocional e 06 (4,8\%) baixa inteligência emocional. Em relação ao desempenho no trabalho, $88(70,4 \%)$ apresentam bom desempenho, $24(19,2 \%)$ desempenho excelente e $13(10,4 \%)$ desempenho ruim. Em conclusão com base nos resultados obtidos, fica evidente que a inteligência emocional tem estreita relação com o desempenho laboral dos profissionais de saúde, uma vez que para alcançar um bom desenvolvimento de suas atividades e poder cumprir os objetivos de sua função devem possuir o emocional. Capacidade de enfrentar os desafios e complicações que surgem ao longo do dia de trabalho. Evidencia-se que a inteligência emocional está relacionada ao desempenho no trabalho, ao se submeter à estatística do teste Tau b de Kendall, com nível de confiança de 95\% e grau de erro de $5 \%$, obteve-se valor $\mathrm{p}=0,01$, o que significa que houve é uma relação estatisticamente significativa entre as variáveis.

Palavras-chave: Inteligência emocional; desempenho laboral; profissional

\section{INTRODUCCIÓN}

$\mathrm{L}$ a capacidad de los individuos para enfrentar situaciones se desarrolla en todos los niveles, y en aspectos personales y profesionales, puesto que sabiendo controlar los sentimientos, emociones propias y de los demás se puede ser capaz de tener un buen desempeño en las actividades cotidianas. Rodríguez (1), en sus diversas investigaciones, menciona que la inteligencia es la capacidad que tienen las personas de poder solventar situaciones o problemas y ser capaz de crear productos que pueden llegar a ser de gran valor, al mencionar que la inteligencia es una capacidad se da a entender que esta puede ser adquirida y desarrollada como una habilidad,, puesto que el autor sugiere que "se puede desarrollar dependiendo de experiencias, educación y el ambiente donde se ha vivido" (1).

Del mismo modo, la inteligencia de una persona está formada por un conjunto de variables como la atención, la capacidad de observación, la memoria, el aprendizaje, las habilidades sociales, etc., que le permiten enfrentarse al mundo diariamente (2). El rendimiento que se obtienen de las actividades diarias depende en gran medida de la atención que el individuo les preste, así como de la capacidad de concentración que manifieste en cada momento. Pero hay que tener en cuenta que, para tener un rendimiento adecuado intervienen muchas otras funciones como, por ejemplo, un estado emocional estable, una buena salud o un nivel de activación normal.

Moreano (2) definieron la Inteligencia Emocional como una Inteligencia Entusiasta considerando al conocimiento con pasión, donde por medio de la IE se aprenda a saber controlar los sentimientos y poder discernir y dirigir nuestro razonamiento (3), Posteriormente Goleman en el año 1998 reformuló esta definición indicando que es la "Capacidad para percibir nuestros propios sentimientos particulares y los de los demás, para estimular y supervisar la emotividad en nosotros mismos y en las conexiones relacionales", e igualmente Rodríguez (1), mantuvo su posición y su definición e inclusive en su Libro "Inteligencia Emocional" menciona que no 
todas las personas poseen un coeficiente intelectual elevado sean exitosos en la vida cotidiana por lo tienden a sentirse decepcionadas, y aquellas personas con un CI más discreto en ocasiones obtienen un progreso bastante bullicioso (1).

Para Extremera (4), la inteligencia emocional se refiere a la interacción adecuada entre Emoción y Cognición, que permite al individuo, un funcionamiento adaptado a su medio (4), la IE es un constructo psicológico bastante complejo e interesante y que aún muchos autores lo definen de diversas formas. Rodríguez (1) menciona que la inteligencia emocional es necesaria y útil para poder determinar la productividad de una empresa y el desempeño laboral de las personas que trabajan en ella, y que la inteligencia emocional posee un conjunto de habilidades (1).

En vista de los grandes cambios actuales, es importante siempre considerar como parte fundamental del desempeño laboral la capacidad de las personas para poder desarrollar sus actividades laborales a diario, teniendo en cuenta como se adapten a la sociedad, como se desenvuelvan personalmente y de manera colectiva, pues es necesario en las empresas tengan personas laborando que sean capaces de poder enfrentar cualquier situación que se presente.

El desempeño laboral es definido por Vásquez (3) como la capacidad que tienen las personas para desarrollar sus actividades,siguiendo las instrucciones del personal que trabaja dentro de las asociaciones, lo que le permite a la persona lograr un trabajo extraordinario y la satisfacción laboral (3). Es importante notar las características individuales de los individuos como son la creatividad, lógica, y capacidad de comprensión de las situaciones lo que permite interpretar que estos aspectos son determinantes en el desenvolvimiento del desempeño laboral (1).

De la misma manera el desempeño laboral presenta una serie de características importantes para su análisis y estudios y se presentan en la Tabla 1.

Tabla 1. Características importantes del desempeño laboral.

CARACTERÍSTICA DEFINICIÓN

Adaptabilidad

Comunicación:

Iniciativa:
Se refiere al mantenimiento de la adecuación en diversas situaciones y con diversas asignaciones, obligaciones e individuos

Se refiere a la capacidad de expresar sus pensamientos con éxito, ya sea en una reunión o de forma independiente. La capacidad de ajustar el dialecto o la redacción a los requisitos del beneficiario (s).

Se refiere a la expectativa de tener un impacto efectivo en ocasiones para lograr objetivos. La capacidad de incitar a las circunstancias en lugar de tolerarlas de manera inactiva. A las medidas que se requieren para cumplir los objetivos más allá de lo requerido 


\section{CARACTERÍSTICA DEFINICIÓN \\ Conocimiento \\ Se refiere al nivel de aprendizaje especializado y / o competente en territorios identificados con su zona de trabajo. La capacidad de mantenerse al día con los avances actuales y los patrones en su entorno general de especialización}

Trabajo en equipo

Estándares de trabajo:

Desarrollo de habilidades

Potencia el diseño del
trabajo

Maximizar del desempeño
Alude a la capacidad de desempeñarse exitosamente en grupos / reuniones de trabajo para lograr los objetivos de la asociación, contribuyendo y creando un dominio agradable que permita el acuerdo

Se refiere a la capacidad de cumplir y superar los objetivos o directrices de la asociación y la capacidad de obtener información para criticar el marco y mejorarlo

Se refiere a la capacidad de desarrollar las aptitudes y capacidades de los colegas, ejercicios de mejora convincentes identificados con los puestos presentes y futuros. Potencia

Se refiere a la capacidad de decidir la mejor asociación y estructura para lograr un objetivo. A la capacidad de reconfigurar adecuadamente el trabajo para amplificar las puertas abiertas para el desarrollo y la adaptabilidad de las personas

La capacidad de establecer objetivos de ejecución / mejora al preparar y evaluar la ejecución en una forma de objetivo.

Fuente: (2)

Así mismo es relevante resaltar la importancia que posee el desempeño laboral donde (1) mencionan que:

Es esencial para el avance autoritario, conocer los propósitos impotentes y sólidos del personal, conocer la naturaleza de cada uno de los asociados, requerido para un programa de elección, mejora administrativa, significado de capacidades y fundamento de una base discerniente e imparcial para compensar la ejecución. Este sistema es imprescindible ya que permite decidir y transmitir a los representantes la manera en que están desarrollando su trabajo y, en un nivel básico, expandir los diseños de cambio. Otra utilización esencial de las evaluaciones para el socio es el avance del cambio de resultados. En este ángulo, se utilizan para transmitir a los representantes cómo están desempeñando sus posiciones o puestos y para proponer los cambios esenciales en la conducta, la mentalidad, las aptitudes o la información. (2).

Igualmente, existen diversos elementos en el desempeño laboral que son necesarios ser evaluados para conocer las condiciones en las que laboran los empleados y como se encuentran ellos (5) Estos elementos son:

- Conocimiento del trabajo: Es el conocimiento obtenido y desarrollado en el lugar de trabajo, el mismo que puede ser adquirido de dos maneras (teórica y práctica).

- Calidad: Chiavenato señala que la calidad del trabajo está relacionada en tres condiciones; ambiente físico, jornada laboral y el ambiente social. 
- Cantidad: Esto refiere a la productividad, la cantidad de producción está enlazada con el grado de motivación que tiene el colaborador al desempeñar sus funciones.

- Exactitud: Refiriéndose a la duración de la jornada laboral y a los beneficios obtenidos por el esfuerzo y desempeño (horas extras y vacaciones).

- Trabajo en equipo: Es necesario establecer buenas relaciones interpersonales adecuadas, pues ello fortalece al ambiente laboral, mejora la producción en grupo y se puede compartir experiencias y habilidades. (3)

En los centros de salud y sobre todo en el área de servicios de hospitalización el estrés y la ansiedad está presente debido al manejo de gran cantidad de pacientes y enfermos que requieren de cuidados $\mathrm{y}$ atenciones por parte de los profesionales de salud, pues los pacientes acuden a estos centros asistenciales con la necesidad de ser atendidos y lograr sentirse mejor en cuanto a su nivel de salud.

El estrés está presente en muchas ocasiones cuando el nivel de responsabilidad en sus labores es bastante alto, aunado a diversos factores como los conflictos, el contacto con pacientes, el clima organizacional entre otros, que generan alarmas en el trabajador en referencia al estrés laboral (6). El estrés laboral siempre está presente en los profesionales de la salud, por ello es sumamente necesario que los empleados esten capacitados para poder enfrentar las situaciones incomodas dentro de la organización, y sean capaces de tener la mejor acttud y el mejor animo para poder desarrollar sus actividades sin complicaciones ni contratiempos (7).
En este sentido se plantea la interrogante: ¿Cuál es la relación que existe entre Inteligencia Emocional $\mathrm{y}$ Desempeño Laboral de los profesionales de la salud de un Hospital de Pucallpa?

Por lo tanto, esta investigación tiene por objetivo establecer la relación entre inteligencia emocional $\mathrm{y}$ desempeño laboral de los profesionales de la salud de un Hospital de Pucallpa. Esto permitirá analizar cómo influye la capacidad que tienen los profesionales de la salud de enfrentar el diario desenvolvimiento de sus funciones en estas instituciones, enfrentando retos, presiones y dificultades durante el desempeño de sus actividades laborales, convirtiéndose en un punto de partida a futuras investigaciones basadas en el estudio de la Inteligencia emocional.

\section{MÉTODO}

$\mathrm{E}$ l tipo de investigación utilizado fue descriptivo, de corte transversal puesto que no se realizó ninguna manipulación de variables y la información recopilada fue en un solo momento (8). En este orden de ideas, se tomaron en cuenta todos los principios bioéticos que rigen este proceso, contando con la aprobación mediante la Constancia № 0007, expedido por el comité de ética de investigación e la Escuela Profesional de Enfermería de la facultad de Ciencias de la Salud de la Universidad Nacional de Ucayali. La población de esta investigación estuvo constituida por 125 profesionales de la salud de un Hospital de Pucallpa conformados por 55 profesionales de sexo masculino y 70 de sexo femenino, estos a su vez están clasificados según su profesión como se muestra en el cuadro 1: 
Cuadro 1. Profesión

\begin{tabular}{lll}
\hline PROFESIÓN & № & \% \\
\hline Enfermero & 58 & 46.4 \\
Médico & 18 & 14.4 \\
Técnico enfermero & 42 & 33.6 \\
Obstetrices & 07 & 5.6 \\
\hline TOTAL & $\mathbf{1 2 5}$ & $\mathbf{1 0 0}$ \\
\hline
\end{tabular}

\section{RESULTADOS Y DISCUSIÓN}

En la Tabla 2 se muestra el nivel de inteligencia emocional que poseen los profesionales de la Salud pertenecientes a los servicios de Hospitalización de un Hospital de Pucallpa.

Tabla 2. Inteligencia emocional de los profesionales de la salud de los servicios de hospitalización de un Hospital de Pucallpa.

\begin{tabular}{lll}
\hline INTELIGENCIA EMOCIONAL & $\mathbf{N}^{\circ}$ & $\mathbf{\%}$ \\
\hline Alto & 35 & $28.0 \%$ \\
Medio & 84 & $67.2 \%$ \\
Bajo & 06 & $4.8 \%$ \\
\hline TOTAL & $\mathbf{1 2 5}$ & $\mathbf{1 0 0 \%}$ \\
\hline
\end{tabular}

Se observó que 84 de los profesionales entrevistados, poseen inteligencia emocional medio el cual representa el $67.2 \%$; 35 de ellos posen inteligencia emocional alto el cual representa el 28.0\%; así mismo seis (6) de ellos presentan inteligencia emocional bajo el cual representa el $4.8 \%$ del total de la muestra.
También en la Tabla 3 se refleja el desempeño laboral que poseen los profesionales de la salud entrevistados pertenecientes a los servicios de hospitalización de un Hospital de Pucallpa.

Tabla 3. Desempeño laboral de los profesionales de la salud de los servicios de hospitalización de un Hospital Pucallpa.

\begin{tabular}{lcc}
\hline DESEMPEÑO LABORAL & $\mathbf{N}^{\circ}$ & $\mathbf{\%}$ \\
\hline Excelente & 24 & 19.2 \\
Bueno & 88 & 70.4 \\
Deficiente & 13 & 10.4 \\
\hline Total & $\mathbf{1 2 5}$ & $\mathbf{1 0 0 \%}$ \\
\hline
\end{tabular}


Es así como en la Tabla 3 se detalló que del total 125 elementos entrevistados, 88 de ellos tienen un desempeño laboral Bueno, el cual representa el 70.4\%; 24 de ellos tienen un desempeño laboral Excelente, el cual representa el 19.2\%; así mismo 13 de ellos presentan Deficiente desempeño laboral, el cual representa el $10.4 \%$ del total de la muestra.
En la Tabla 4 se refleja la relación existente entre la inteligencia emocional y el desempeño laboral de los profesionales de la salud entrevistados pertenecientes a los servicios de hospitalización de un Hospital de Pucallpa.

Tabla 4. Relación entre inteligencia emocional y desempeño laboral de los profesionales de la salud de los servicios de hospitalización de un Hospital de Pucallpa.

\begin{tabular}{lcccc}
\hline $\begin{array}{c}\text { INTELIGENCIA } \\
\text { EMOCIONAL }\end{array}$ & EXCELENTE & BUENO & DEFICIENTE & TOTAL \\
\hline Alto & 24 & 11 & 00 & 35 \\
Medio & 00 & 76 & 08 & 84 \\
Bajo & 00 & 01 & 05 & 06 \\
\hline Total & 24 & 88 & 13 & 125 \\
\hline
\end{tabular}

En la Tabla 4, se aprecia que, del total de 125 elementos entrevistados, con respecto a la relación entre inteligencia emocional y desempeño laboral, 84 de ellos poseen inteligencia emocional medio del cual la mayoría 76 tienen un desempeño laboral bueno; 35 de ellos poseen inteligencia emocional alto $\mathrm{y}$ mayormente 24 tienen un desempeño laboral excelente; así mismo 06 de ellos poseen inteligencia emocional bajo y donde prima 05 el desempeño laboral deficiente.

En consecuencia, se obtiene que el nivel de inteligencia emocional es medio en más de la mitad (67.2\%); con tendencia a alto, lo que significa que los profesionales de la salud desarrollan un manejo regular de diversas situaciones en torno a su ambiente laboral, con desempeño Bueno, lo que implica que no desarrollan a cabalidad las funciones asignadas como profesional de la salud, situación que depende en gran medida de voluntad política para su mejora.

\section{Comprobación de hipótesis}

\section{Estadístico de prueba}

Para su comprobación de sometido al estadístico de prueba Tau b de Kendall por ser ambas variables de tipo categórico ordinal, con un nivel de confianza del $95 \%$ y grado de error del 5\%; obteniendo un valor $\mathrm{p}$ (significancia) $=0.01$, por tanto, se acepta la hipótesis de investigación y se rechaza la hipótesis nula.

\section{Hipótesis de investigación (Hi)}

Existe relación directa y significativa entre inteligencia emocional y desempeño laboral de los profesionales de la salud de un Hospital de Pucallpa. 


\section{Hipótesis Nula (Ho)}

No existe relación directa ni significativa entre Inteligencia Emocional y Desempeño Laboral de los profesionales de la salud de un Hospital de Pucallpa.

\section{CONCLUSIONES}

$\mathrm{E}$ $n$ base a los resultados obtenidos es evidente que la inteligencia emocional tiene una estrecha relación con el desempeño laboral de los profesionales de la salud puesto que para lograr un buen desenvolvimiento de sus actividades y poder cumplir con los objetivos de su cargo debe poseer la capacidad emocional de sobrellevar los retos y complicaciones que se le susciten a lo largo de su jornada laboral.

Se evidencia que la inteligencia emocional se relaciona con el desempeño laboral, al someter al estadístico de prueba de Tau b de Kendall, con un nivel de confianza del $95 \%$ y grado de error del $5 \%$, se obtuvo un valor $\mathrm{p}=0.01$, lo que significa que existe relación estadísticamente significativa entre las variables.

\section{REFERENCIAS BIBLIOGRÁFICAS}

1. Rodríguez L. Inteligencia emocional y su relación con el desempeño laboral del personal de salud del hospital regional docente de Trujillo-Perú 2008-2009. Trujillo, Perú, 2011

2. Moreano N. La inteligencia emocional y el desempeño laboral del profesional de enfermería del Centro Médico Naval "Santiago Távara" Callao. 2018. Lima, Perú, 2018

3. Vásquez E, Vásquez PD. Inteligencia Emocional y su relación con el desempeño laboral en profesionales de salud de la microred de Cajamarca-Categoría I3. 2019. Cajamarca, Perú, 2019

4. Extremera N, Fernández P. El papel de la inteligencia emocional en el alumnado: evidencias empíricas. Revista Electrónica de Investigación Educativa, 20046 (2)

5. Jiménez M, López E. Inteligencia emocional y rendimiento escolar: estado actual de la cuestión. Revista Latinoamericana de psicología, 41(1). 2009: 69-79

6. Molina MC. Influencia de la Inteligencia Emocional en el Desempeño Laboral. Guatemala, 2020

7. Mamani A, Obando R, Uribe A, Vivanco M. Factores que desencadenan el estrés y sus consecuencias en el desempeño laboral en emergencia. Rev Per Obst Enf 3(1). 2007: 50-57

8. Palella Stracuzzi S, Martins Pestana F. Metodología de la investigación cuantitativa Caracas, Venezuela: FEDUP|EL, Fondo Editorial de la Universidad Pedagógica Experimental Libertador; 2010

Conflicto de intereses. El autor declara que no existe conflicto de intereses para la publicación del presente artículo.

Financiamiento. Autofinanciamiento Agradecimiento. No declaran 